\title{
Effect of Facilitating Reciprocal Inhibition of Ankle Flexors on Muscle Activation Pattern in Sit to Stand Movement in Stroke -Case Study-
}

\author{
Woo-Nam Chang ${ }^{1}$, Young-Dong Kim², Kyoung-Bo Lee ${ }^{3}$, Jeon-Hye Joo ${ }^{4}$, Sang-Mi Jung ${ }^{* 5}$ \\ ${ }^{1}$ Dept. of Physical Therapy, College of Health Welfare, Yong-In University \\ ${ }^{2}$ Dept. of Physical Therapy, Yuseong Hankajok Hospital \\ ${ }^{3}$ Dept. of Physical Therapy, Catholic University, St. Vincent's Hospital \\ ${ }^{4}$ Dept. of Physical Therapy, Bobath Memorial Hospital \\ ${ }^{*}$ Dept. of Occupational Therapy, Sangji Youngseo College
}

\begin{abstract}
Purpose The aim of this study was to determine the effects of facilitating reciprocal inhibition of ankle dorsi-Plantar flexors on leg muscle activation patterns during the sit to stand movement. Methods Fifteen patients with hemiplegia were randomly recruited from $\mathrm{Y}$ hospital. Subjects stood up from an armless plinth with a comfortable and self-paced speed. Onset-offset times of leg muscles during the sit to stand movement were measured using surface electromyograph and a force platform. Results The mean onset time of muscle activation in paretic tibialis anterior (TA) was significantly earlier after treatment $(\mathrm{p}<.05)$, and soleus (SOL), which showed a significant delay $(\mathrm{p}<.05)$ in paretic side. However, there was no significant difference between pre- and post-treatment in rectus femoris (RF) and biceps femoris (BF) ( $>$.05) in paretic side. There were significant differences between pre- and post-treatment in onset time of both paretic and non-paretic leg muscle $(\mathrm{p}<.05)$. The recruitment order of muscle activity in pre-treatment was in sequence of RF, SOL, then followed by TA activation. In post-treatment, it was in order of RF activation, TA, BF and then SOL activation. Conclusion It was found that the therapeutic exercise to facilitate the intrinsic muscle and antagonistic activity of SOL in paretic foot and leg made significant effects on enhancing reciprocal inhibition for efficient postural control.
\end{abstract}

Key words Ankle function, Muscle activation pattern, Reciprocal inhibition, Sit to stand movement

Corresponding author Sang-Mi Jung (otjsm@hanmail.net)

Received date 18 January 2018

Revised date 10 February 2018

Accepted date 21 February 2018

\section{Introduction}

The sit-to-stand movement is a precondition for independent locomotion and activities of daily living. ${ }^{1)}$ During the sit-to-stand movement, a stable sitting posture is converted into a relatively unstable standing posture $^{2)}$ and it is a transitional movement that connects sitting posture to walking. Healthy persons can easily and naturally perform this movement but hemiplegic patients due to stroke show difficulties in performing this movement. ${ }^{3)}$

In the lower limb muscle activation pattern in the sit-to-stand movement, the tibialis anterior (TA) muscle

doi : http:dx.doi.org/10.17817/2018.02.13.111233 is activated first to move the body's center of gravity forward and plays central roles for the timing and pattern of ankle muscle activation. ${ }^{4,5)}$ Almost simultaneously with the activation of the TA, the rectus femoris (RF) muscle is activated to work as a knee extensor to provide the stability of the knee and the hip joint. After the activation of the TA and the RF, the biceps femoris (BF) muscle is activated. The BF maintains the stability of the knee when the hip has been taken off the bed and helps the extension of the hip joint. ${ }^{6}$ The soleus (SOL) muscle is activated last and plays the role of adjusting the speed of knee extension when the maximum ankle flexion is converted into extension in the stage of the sit-to-stand movement and controlling the posture so that stable stand- 
ing postures can be maintained.

Normally, the TA e and the SOL are reciprocally inhibited while flexion and extension are attempted." Reciprocal inhibition refers to alternating occurrence of inhibition and facilitation of an agonistic and an antagonistic muscle and this function does not properly operate in some pathological conditions. For instance, if the activity of the SOL increases abnormally, muscle activity will become hard even if the TA is normal. ${ }^{7,8)}$

A movement that is the most frequently used in functional movement assessment performed at clinics is the sit-to-stand movement. The sit-to-stand movement is also used for therapeutic purposes such as muscle strengthening, improvement of postural control, and functional activities. Therefore, the sit-to-stand movement and the stand-to-sit movement are diversely performed in assessments and therapeutic approaches. However, studies related to the foot investigated changes in the order and duration of muscle activation according to foot positions in healthy persons and hemiplegic patients.

The purpose of this study was to investigate the effects of the exercises which are to active intrinsic foot muscles and facilitate reciprocal inhibition of ankle muscles on the neurologic factors that determine muscle balance during the sit-to-stand movement.

\section{Materials and Methods}

\section{Study subjects}

The subjects of this study were fifteen hemiplegic patients ( 8 males and 7 females) after stroke at $Y$ hospital who fully and sufficiently understood and agreed to the purpose of the study. After being evaluated, the patients were provided with treatment intervention that facilitated reciprocal inhibition of the ankle dorsi-plantar flexors for 30 minutes. After the therapeutic intervention, the patients could take a rest in a supine posture for 10 minutes and reevaluation was performed thereafter. The inclusion criteria were as follows: 1) no past history of respiratory, 2) no past musculoskeletal disease 3) no perceptual disturbance such as hemi-neglect, 4) capable of independent sit-to-stand movements, and 5) at least 24 points in the Korean version of the mini-mental state examination test (K-MMSE) (Table 1).

Table 1. General characteristics of the subjects $(\mathrm{N}=15)$

\begin{tabular}{|c|c|c|c|}
\hline Variable & & $\mathrm{n}(\%)$ & Mean $\pm S D$ \\
\hline Age (yrs) & & 15 & $47.86 \pm 16.73$ \\
\hline \multirow[t]{2}{*}{ Sex } & Male & $9(60)$ & \\
\hline & Female & $6(40)$ & \\
\hline Body weight (kg) & & & $61.33 \pm 8.37$ \\
\hline Body height (cm) & & & $165.13 \pm 5.89$ \\
\hline \multirow[t]{2}{*}{ Type of stroke } & Hemorrhage & $8(53)$ & \\
\hline & Infarction & $7(47)$ & \\
\hline \multirow[t]{2}{*}{ Side affected } & Rigtht & $9(60)$ & \\
\hline & Left & $6(40)$ & \\
\hline \multirow[t]{2}{*}{ Duration of stroke (mos) } & $1-12$ & $10(67)$ & $8.46 \pm 8.87$ \\
\hline & Above 12 & $5(33)$ & \\
\hline \multirow[t]{3}{*}{ MBI score } & Severe & $5(34)$ & $64.26 \pm 23.58$ \\
\hline & Moderate & $2(13)$ & \\
\hline & Mild & $8(53)$ & \\
\hline Spasticity & G0-2 & 15 & \\
\hline \multirow[t]{2}{*}{ Sensory function } & No impairment & $5(33)$ & \\
\hline & Impairment & $10(67)$ & \\
\hline
\end{tabular}

Mean \pm SD, Mean \pm Standard Deviation; MBI, Modified Barthel Index 


\section{Study method}

Wireless surface electromyography (Aurion, Italy) was used to measure the onset times of muscle activation of four muscles that act importantly in the sit to stand movements ${ }^{10)}$, that is, the TA, SOL, RF, and BF (Fig 1-A). A force platform from Kistler Co. was used to obtain kinetic data from movements occurred in sitting postures. The data, obtained through the surface EMG and the force platform, were analyzed using a QTM system made by Qualisys Co. EMG and force platform signals were synchronized using an analog-digital converter. (Fig 1-B, C)

\section{1) Study procedure}

For the same study environments of the individual subjects considering their physical conditions, a height-adjustable treatment bed without any armrest or backrest was used. Each subject placed two feet on the force platform in parallel with each other at $10 \mathrm{~cm}$ apart based on the medial planes of the feet. As a start posture, each subject maintains $10^{\circ}$ flexion of the ankle and $100^{\circ}$ flexion of the knee joint and placed the arms on both sides. The subjects were instructed to stand up comfortably at the speed at ordinary times. The subjects performed the sit to stand movement at the command 'Stand up' after a preparatory command to prepare. This movement was repeated three times and the average value was obtained. ${ }^{10,11)}$ After the baseline evaluation, the subject took a rest for 10 minutes in a supine posture after the inter- vention, and reevaluation was performed in the same method after the rest. (Figure 2). The start of the movement was defined as the section from the point at which the vertical ground reaction force began to decrease to the point showed the minimum value. The movement execution stage was defined as the section from the point at which the vertical ground reaction force began to increase to the point reached the peak. The final stationary stage was defined to the point at when the ground reaction force was stabilized. The stages from the start to the end of movement were normalized to analyze the time of onset of muscle activation and ground reaction force data. $^{10)}$

2) Therapeutic intervention

(1) Realignment of the feet and ankles and facilitation of muscle activation

(1) For selective recovery of the lengths of the TA and SOL and muscle activation, the treatment bed should be high enough so that both feet are held away from the ground before beginning the treatment. As for the position of the foot to be held, the medial side of the foot including the big toe should be maintained stable with one hand and the lateral side of the foot including the little toe should be held with the other hand of the physical therapist (A). Sensory input should be provided between the abductor digiti minimi muscle and the metatarsal bone through movements by
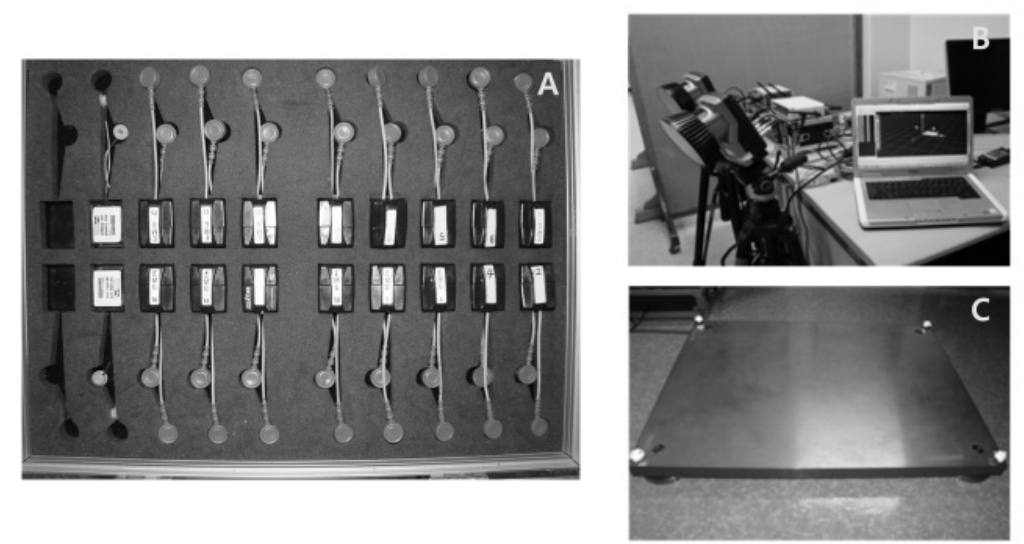

Figure 1. Zero-Wire EMG(A), QTM system(B) and force platform(C)

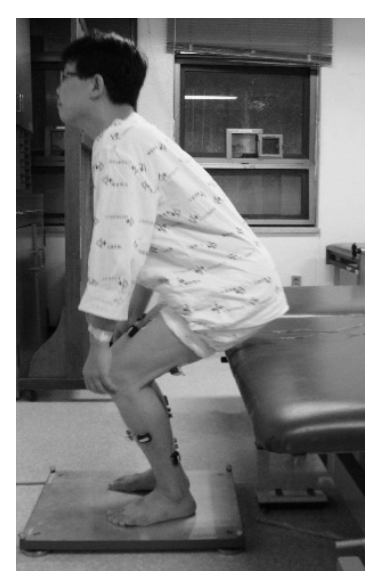

Figure 2. Data collection during sit to stand movement 

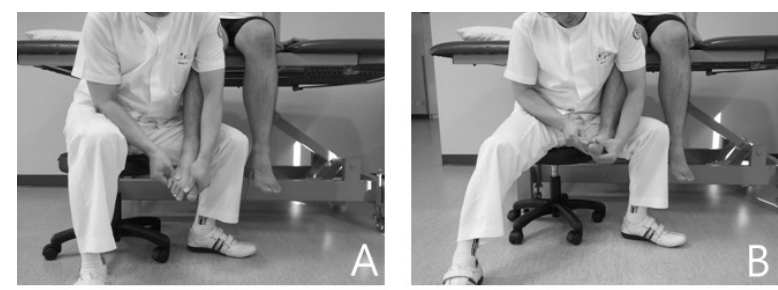

changes in the intrinsic muscles between the sole and toes (B). ${ }^{8)}$

(2) The therapist should hold the fore part of the foot with one hand and the heel of the foot with the other hand (C). The therapist should make the subject lower the heel slowly toward the floor as the heel pushes down with the eccentric contraction of the SOL in the sit-to-stand movement (D).

(2) Movement composition and execution facilitation method

(1) The treatment bed should be lowered so that the feet can be touched on the ground before starting the treatment. The subject should sit on the treatment bed with the feet and heels placed flat on the floor. The therapist should support the heel of a foot with one hand and hold the top of the foot with the other hand to facilitate the eccentric contraction of the SOL (A). The therapist should reinforce the interaction between the TA and SOL through repetitive lifting and putting down of the heel (B).

(2) The therapist should hold the top of the foot close to the ankle with one hand and hold the lower part of the tibia with the other hand. The therapist should softly press the foot backward so that the
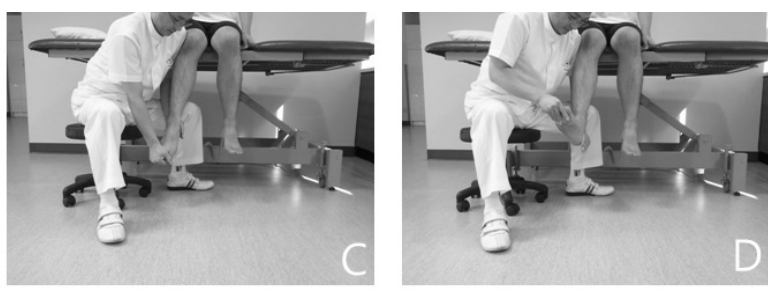

heel gradually comes into tight contact with the floor. The therapist should move the foot backward with the heel maintained in contact with the floor to lengthen the SOL (C). The therapist should instruct the subject to keep the heel in tight contact with the floor to achieve the maximum contact. The therapist should sit in front of the subject and place both hands on the knee extensors of the subject and press the knee extensors down toward the heel (D).

\section{4) Statistical analysis}

The means and standard deviations of the general characteristics of the subjects were obtained through descriptive statistics and the normality of the measured data was identified through a 1-sample KolmogorovSmirnov analysis. Paired t-tests were conducted to analyze changes in muscle activities through the therapeutic intervention and the one-way repeated ANOVA was used to analyze the order of muscle activation of the lower limbs before and after the treatment. The data were statistically processed using the SPSS 12.0 program and the significance level was set to 0.05 .
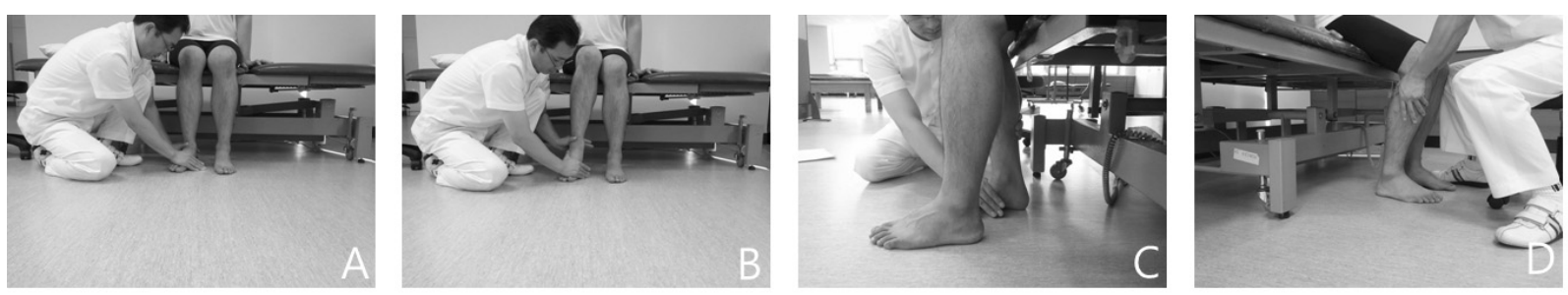


\section{Results}

\section{Changes in onset time of muscle activity}

Regarding changes in both lower limb muscle activity onset times between before and after the treatment, among paretic side lower limb muscles, the TA showed significantly earlier onset after the treatment (25.27\%), while the SOL showed delayed onset $(22.86 \%)(\mathrm{p}<0.05)$. The RF and BF showed delays in average values, but the differences were not statistically significant $(\mathrm{p}>0.05)$. Non-paretic lower limb muscles showed no significant change in onset time of muscle activity between pre- and post-treatment $(\mathrm{p}>0.05)$.

\section{Changes in recruitment order of lower limb muscle activity}

Recruitment orders were significant between the paretic side $T A$ and $R F$ and between the paretic side SOL and RF before the treatment $(\mathrm{p}<0.05)$. Therefore, regarding the recruitment order of paretic lower limb muscle activity before the treatment, the RF was activated first followed by the SOL and TA in order of precedence. The recruitment order of paretic muscle activity in the post-treatment was significant and the TA was activated first followed by the SOL, the RF, and the $\mathrm{BF}(\mathrm{p}<0.05)$. The recruitment order of non-paretic lower limb muscle activity was statistically significant $(\mathrm{p}<0.05)$. The recruitment order between pre-and post-treatment were the same and the TA was activated first followed by the $\mathrm{RF}$, the $\mathrm{BF}$, and the SOL.

Table 2. Variations of muscle onset time between pre- and post-treatment during the sit to stand movement (n=15)

$($ Value $=$ Mean \pm SD $)$

\begin{tabular}{ccccccc} 
Muscle & \multicolumn{3}{c}{ Paretic Side } & \multicolumn{3}{c}{ Non-paretic Side } \\
& Pre & Post & $t^{*}$ & Pre & \multicolumn{2}{c}{ Post } \\
TA & $49.26 \pm 20.64$ & $36.81 \pm 14.14$ & $3.01^{*}$ & $16.08 \pm 12.01$ & $21.02 \pm 8.77$ & -1.50 \\
SOL & $44.00 \pm 5.95$ & $54.06 \pm 17.64$ & $-2.28^{*}$ & $49.82 \pm 13.59$ & $51.79 \pm 18.07$ & -0.44 \\
RF & $30.39 \pm 8.06$ & $35.93 \pm 10.80$ & -1.53 & $25.36 \pm 7.44$ & $29.45 \pm 8.18$ & -1.20 \\
BF & $35.29 \pm 13.67$ & $36.59 \pm 14.54$ & -0.34 & $38.72 \pm 10.31$ & $38.67 \pm 12.11$ & 0.01 \\
\hline
\end{tabular}

$: p<.05 ;$ Mean $\pm \mathrm{SD}$, Mean \pm Standard deviation

Note: TA, tibialis anterior muscle; SOL, soleus muscle; RF, rectus femoris muscle; BF, biceps femoris muscle
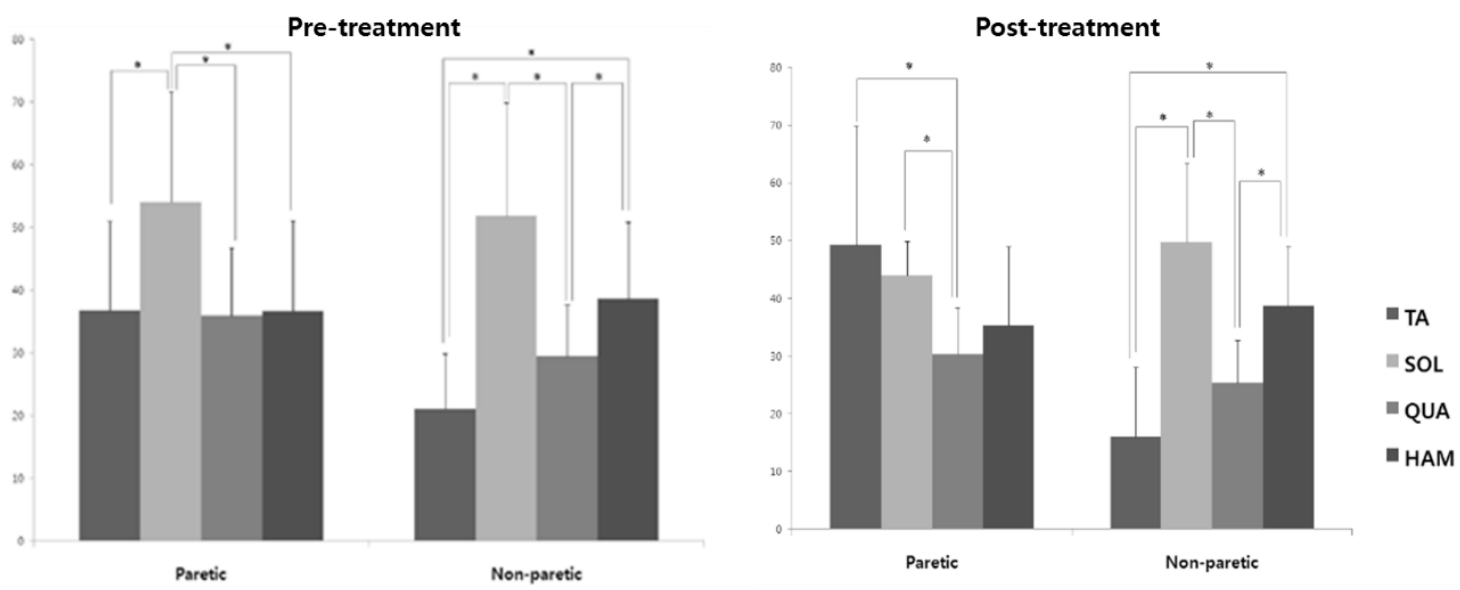

Figure 3. Variations of the recruitment order of muscle activation between pre- and post-treatment during the sittostand movement. 


\section{Discussion}

Clinically, patients with neurological damage have a reduced ability to maintain a sitting posture without any backrest or to shift their weight in sitting postures. Therefore, they become to use compensatory postural strategies. ${ }^{8)}$ The use of compensatory postural strategies means making excessive movement patterns with biased recruitment, that is, one-sided muscle contraction. According to Sahrmann (1992), shortened muscles are more easily mobilized than extended contralateral antagonists, resulting in increases in the muscle tension of the shortened muscles. ${ }^{12)}$ In a study conducted by Cheng et al. (2004), the TA showed no activation or very low activity during the sit to stand movements of hemiplegic patients which is consistent with the results of another study indicating that hemiplegic patients showed asymmetric muscle activities of two lower limbs due to excessive non-paretic side TA activation. ${ }^{10)}$

Raine et al. (2009) reported that the shortening and weakening of the SOL as an antagonistic muscle restricted dorsi-flexion resulted in excessive medial elevation. ${ }^{8)}$ The ineffective ankle alignment as such was attributed to the compensatory activation of the TA. The abovementioned results related to the TA are considered attributable to the treatment method in this study that facilitated the activation of the lateral elevator while maintaining the length of the muscle in order not to induce excessive contraction of the TA, which is a medial elevator, thereby reinforcing the antagonist which is the SOL. The pre-treatment muscle activity of the paretic side SOL was $73 \%$ of that of the non-paretic side SOL, but decreased to $43 \%$ after the treatment. This can be considered attributable to the fact that the action potential of the uncontrolled excessive activity of the TA before the treatment was reduced through the recovery of the antagonistic muscle and the synergist after the treatment.

Regarding lower limb muscle activity onset time, the paretic side TA showed significantly earlier activity onset time after the treatment compared to that before the treatment while the SOL showed delayed activity onset time with a statistically significant difference. In the study conducted by Goulart and Valls-Sole (2001) on the reciprocal inhibition of the TA and SOL in healthy persons, it was reported that information coming in from the periphery was suppressed for a certain time for the antagonism of the soleus muscle during the sit-to-stand movements and that the foregoing facilitated the activation of the TA, which is an agonist, through ankle stability. ${ }^{5)}$ In normal study subjects, the SOL is activated last among lower limb muscles and is activated after the hip taking off during the sit-to-stand movement. $^{5) 6}$

The result indicating that the SOL is activated earlier than the TA before the treatment was similar to the results of the study conducted by Camargos et al. (2009), in which changes in the muscle activity and muscle activity onset time according to changes in foot positions during hemiplegic patients' sit-to-stand movement were analyzed, indicating that the SOL was activated before hip taking off regardless of foot positions. ${ }^{13)}$ Therefore, the significant differences in the TA and SOL activity onset times can be said to mean the enhancement of the ability for reciprocal inhibition of dorsi-flexion and plantar flexion.

The recruitment order of muscle activation was statistically significant on both paretic and non-paretic lower limb muscles. The recruitment order of muscle activation in pre-treatment was the RF, SOL, and TA. Therefore, the SOL was activated earlier than the TA. The order of muscle activation as such becomes to disturb the movement of the center of gravity toward the front of the feet and disturb the sufficient extension of the hip joint with early extension of the knee joint. ${ }^{8)}$ After the treatment, the TA and SOL showed significant differences in the recruitment order. The delayed activation of the SOL can be regarded to contribute to postural control at vertical acceleration and deceleration time, that is, during the extension of the lower limb joints with the enhancement of the antagonism of the plantar flexor.

Given the abovementioned results, the fact that the treatment method that facilitates the reciprocal inhibition of dorsi and plantar flexion of the ankle in patients with hemiplegia due to stroke improves the muscle activity patterns of lower limb muscles on both 
sides could be identified and it could be seen that such results would affect improvement of the sit-to-stand movement efficiently.

\section{Conclusion}

Through the results of this study, it could be identified that the intervention used in this study reinforces the muscle activity patterns of both side lower limb muscles and it could be concluded that such results would affect stable postural control during the sit-to-stand movement. Therefore, we believe that the treatment would prevent changes in the neurologic reciprocal inhibition of the feet and ankles and biomechanical alignments and can provide therapeutic benefits to hemiplegic patients in need.

\section{References}

1. Lomaglio MJ, Eng JJ. Muscle strength and weight-bearing symmetry relate to sit to stand performance in individuals with stroke. Gait Posture. 2005;22:126-31.

2. Mark MKY, Levin O, Mizrahi J, et al. Joint torques during sit-to-stand in healthy subjects and people with parkinson's disease. Clin Biomech. 2003;18:197-206.

3. Carr JH, Shepherd RB. Neurological Rehabilitatin: Optimizing Motor Perforamance. Oxford, ButterworthHeinmann. 2003.
4. Goulart F, Valls-Sole J. Patterned electromyographic activity in the sit to stand movement. Clin Neurophysiol. 1999;110(9):1634-40.

5. Goulart F, Valls-Sole J. Reciprocal changes of excitability between tibialis anterior and soleus during the sit to stand movement. Exp Brain Res. 2001;158(1):18-27.

6. Khemlani MM, Carr JH, Crosbie WJ. Muscle synergies and joint linkages in sit to stand under two initial foot positions. Clin Biomech. 1999;14:236-46.

7. Gjelsvik B. The Bobath concept in adult neurology. Stuttgart: Thieme. 2008.

8. Raine S, Meadows L, Lynch-Ellerington M. Bobath concept. Oxford: Wiley-Blackwell. 2009.

9. Cheng PT, Liaw MY, Wong MK, et al. The sit to stand movement in stroke patients and its correlation with falling. Arch Phys Med Rehabil, 1998;79:1043-6.

10. Cheng PT, Chen CL, Wong MK, et al. Leg muscle activation patterns of sit to stand movements in stroke patients. Am J Phys Med Rehabil, 2004;82:42-7.

11. Chou SW, Wong AMK, Leong CP, et al. Postural control during sit to stand and gait in stroke patients. Am J Phys Med Rehabil, 2003;82(1):42-7.

12. Sahrmann SA. Diagnosis and treatment of movement impairment syndromes. St. Louis, MO: Mosby. 2002.

13. Camargos AC, Rodrigues-de-Paula-Goulart F, Teixeira-Salmela LF. The effects of foot position on the performance of the sit to stand movement with chronic stroke subjects. Arch Phys Med Rehabil, 2009;90:314-9. 